\title{
CURRÍCULO NA EDUCAÇÃO SUPERIOR: QUE- SITOS PARA A FORMAÇÃO EM PRODUÇÃO CULTURAL
}

\author{
ELISA LÜBECK \\ UNIVERSIDADE FEDERAL DO PAMPA \\ SÃO BORJA, RIO GRANDE DO SUL, BRASIL \\ E-MAIL: ELISATERRA@UNIPAMPA.EDU.BR \\ MARIA CRISTINA PANSERA-DE-ARAÚJO \\ UNIVERSIDADE REGIONAL DO NOROESTE \\ IJUÍ, RIO GRANDE DO SUL, BRASIL \\ E-MAIL:PANSERA@UNIJUI.EDU.BR
}

HTTP://DX.DOI.ORG/10.5902/2316882X22462 


\section{CURRÍCULO NA EDUCAÇÃO SUPERIOR: QUESITOS PARA A FOR- MAÇÃO EM PRODUÇÃO CULTURAL}

Resumo: O presente artigo tem por objetivo compreender os processos de formação e profissionalização no campo da cultura, em especial sobre as relações e interações com o cenário social contemporâneo na organização e implantação de currículos de graduação em produção cultural. Visto que, os currículos dos cursos são (de)marcados pela constante interação com o cenário social, em que emerge uma nova perspectiva da cultura, interligada com os processos de desenvolvimento econômico, político e social. Dessa forma, delimitou-se o contexto de atuação dos profissionais de produção cultural, observando o que é essencial na formação a fim de estabelecer um perfil adequado para atuação no sistema de organização da cultura - um perfil que valorize e desenvolva a autonomia dos sujeitos. Sendo assim, observou-se que os profissionais devem ter um perfil multidisciplinar, capaz de trabalhar com a incerteza, reposicionando-se no debate sobre a cultura, a alteridade, a identidade, a cidadania, a diversidade e a interculturalidade.

Palavras-chave: Cultura; Educação; Formação acadêmico-profissional; Produção cultural.

\section{PLAN DE ESTUDIOS DE EDUCACIÓN SUPERIOR: REQUISITOS PARA LA FORMACIÓN EN PRODUCCIÓN CULTURAL}

Resumen: Este artículo tiene como objetivo comprender los procesos de formación y profesionalidad en el campo de la cultura, en particular sobre las relaciones e interacciones con el escenario social contemporáneo en la organización y ejecución de los planes de estudio universitarios en producción cultural. Puesto que los planes de estudio están marcados por la constante interacción con el escenario social en que surge una nueva perspectiva de la cultura, relacionada con los procesos de desarrollo económico, político y social. Así, delimitado por el contexto del desempeño de la producción cultural, viendo lo que es esencial en la formación con el fin de establecer un perfil adecuado para el funcionamiento del sistema de organización de la cultura, que desarrolla la autonomía del sujeto. Así, se observó que los profesionales deben tener un perfil multidisciplinario, capaz de trabajar con incertidumbre, reposicionándose en el debate sobre cultura, alteridad, identidad, ciudadanía, diversidad e interculturalidad.

Palabras Clave: Cultura; Educación; Formación académica y profesional; Producción cultural.

Rev.Cad. Comun. Santa Maria, v.20, n.2, art 3, p.2 de 19, maio/ago.2016 


\title{
CURRICULUM IN HIGHER EDUCATION: REQUIREMENTS FOR TRAI-
} NING IN CULTURAL PRODUCTION

\begin{abstract}
The following article aims to understand the processes of formation and professionalization in the field of culture, particularly, concerning relations and interactions with the contemporary social scenario in the organization and implementation of undergraduate syllabus in cultural production. Since the syllabus of the majoring is marked by constant interaction with the social setting in which a new perspective of culture emerges, interconnected with the processes of economic, political and social development, I have delimited the context of cultural production professionals, taking into account what is essential in the formation process in order to establish an appropriate profile for operations in the organizational system of culture, a profile that values and develops the autonomy of the subject. Professionals should have a multidisciplinary profile, capable of working with uncertainty, repositioning in the debate about culture, otherness, identity, citizenship, diversity, and interculturalism.

Keywords: Academic-professional formation; Culture; Cultural production; Education.
\end{abstract}




\section{INTRODUÇÃO}

O Brasil passou por um momento muito especial de valorização da cultura e de construção e efetivação de políticas públicas de cultura, um momento em que todos, sejam agentes, produtores, público em geral ou gestores, buscaram participar e interferir nos processos. Um momento em que voltaram à cena as mais variadas manifestações culturais locais. Dessa forma, cresceram as demandas por formação e especialização dos produtores e agentes culturais em todos os níveis, em busca de uma autonomia cultural.

Este artigo tem como objetivo refletir sobre os processos de formação e profissionalização no campo da cultura no Brasil, em especial, na implantação de currículos de graduação para a formação na área de produção cultural, e suas relações com o cenário social contemporâneo, compreendendo que, "formar é muito mais do que puramente treinar o educando no desempenho de destrezas" (FREIRE, 2011, p.16), mas permitir ao homem tornar-se sujeito, construir-se e fazer a cultura e a história. Nesse sentido, observou-se que os currículos dos cursos de graduação em produção cultural constituíam-se numa perspectiva de hibridismo cultural ${ }^{6}$ e interculturalidade ${ }^{7}$ (de)marcados por interações com o cenário social contemporâneo, em que a formação era fruto de uma nova perspectiva de cultura, interligada aos processos de desenvolvimento econômico, político, ambiental e social.

A formação dos profissionais da área da cultura ocorreu no cotidiano do trabalho, e somente a partir de 1980, com a criação das instituições públicas de cultura e com a legislação de incentivo fiscal e financiamento do setor cultural, é que se iniciou a trajetória de reconhecimento da profissão. Para Cunha (2013), é essencial um equilíbrio entre a formação teórica e a prática para que as matrizes curriculares de produção cultural se adequem as demandas contemporâneas da atuação profissional.

6 O conceito de hibridismo não deve limitar-se a descrever as misturas interculturais, mas deve ser "útil para interpretar as relações de sentido que se reconstroem nas misturas" (GARCÍA CANCLINI, 2008, p.XXIV).

$7 \quad$ García Canclini (2007b) entende a interculturalidade como a diversidade de culturas em confrontação e entrelaçamento, com relações e trocas em um mundo globalizado.

Rev.Cad. Comun. Santa Maria, v.20, n.2, art 3, p.4 de 19, maio/ago.2016 
Ao trabalhar o perfil e o campo de atuação do gestor cultural, uma profissão relativamente nova, é preciso analisar também o seu processo formativo, considerando como base de sustentação teórica conhecimentos multidisciplinares, que deve estabelecer uma relação entre as questões artísticas e culturais associadas aos conhecimentos sociológicos, antropológicos e políticos, bem como aos conhecimentos mais técnicos da comunicação, economia, administração e direitos aplicados à esfera cultural. (CUNHA, 2013, p.24)

Para isso, é essencial nos cursos de graduação o debate sobre os conceitos e usos contemporâneos da cultura, pois eles exigem a construção de um perfil profissional diferenciado, "capaz de olhar de forma estratégica e sensível o seu campo de atuação, capacitado para ocupar funções novas que surgem no setor cultural" (CUNHA, 2013, p.7).

Para Anastasiou (2005), os currículos devem permitir ao discente construir um quadro teórico-prático global, superando a fragmentação, tornando-o capaz de adequar-se a uma realidade dinâmica, desenvolvendo a interpretação, espontaneidade, auto-organização, criatividade, evolução e o reconhecimento do contexto histórico, social e político no qual está inserido.

Este olhar sobre a profissão e sobre os currículos de graduação em produção cultural, tem como objetivo acompanhar as tendências desta área no Brasil, que exige profissionais cada vez mais conscientes da realidade sociocultural, política e econômica. Profissionais competentes para atuarem em uma área em constante evolução, desenvolvendo a capacidade de aprimoramento, criatividade e formação continuada, observando que os currículos devem ser estudados dentro de seu contexto social, uma vez que são historicamente situados e culturalmente determinados (VEIGA, 2002).

\section{CURRÍCULO NA EDUCAÇÃO SUPERIOR}

Nos últimos anos, as discussões sobre currículo vêm assumindo maior importância, principalmente em função de alterações nas propostas curriculares oficiais. Associada a essa centralidade, observou-se uma multiplicidade de referências para o campo do currículo, tornando difícil a sua delimitação. São especialmente relevantes as transformações que ocorreram na sociedade como a globalização, a mundialização da cultura, a redução

Rev.Cad.Comun. Santa Maria, v.20, n.2, art 3, p.5 de 19, maio/ago.2016 
das distâncias espaço-temporais, a substituição das identidades-mestras, baseadas na ideia de nação, por identidades locais muito mais plurais. Nesse contexto, o currículo volta-se para temáticas bastante diversas.

O currículo é assim uma forma de representação que se constitui como sistema de regulação moral e de controle. Tanto é produto das relações de poder e identidades sociais, quanto seu determinante (p.28). [...] Em 1992, as ideias de um currículo com uma base comum nacional e com eixos curriculares articuladores das experiências de formação deram origem a uma proposta curricular na Universidade Federal Fluminense, objeto de vários textos discutidos em reuniões de curriculistas na primeira metade da década. São esses textos que começam a incorporar o conceito de conhecimento em rede para a discussão do currículo (LOPES e MACEDO, 2010, p.33).

Para Lopes e Macedo (2010), a marca do currículo no Brasil, a partir da década de 90, foi o hibridismo, evidenciando uma preocupação constante com os eixos curriculares como espaços de discussão coletiva, englobando uma diversidade de tendências e influências, fundamentadas "na filosofia do sujeito, na filosofia da consciência e na valorização do conhecimento como produtor de sujeitos críticos e autônomos" (LOPES e MACEDO, 2010, p.47). Outro aspecto marcante foi a associação da educação e do currículo a processos culturais amplos com transformações, mudanças, singularidades e incertezas, característicos da nossa sociedade.

Um currículo da diferença é, assim, todos os currículos que nos sentimos convocados a criar, quando abrimos o jornal todo dia, neste preciso momento, no mundo, na história, e ficamos desassossegados, desconcertados, desalinhados, desarranjados com a existência dos diferentes e suas diferenças, a quem nos compete educar. Além disso, ele é cada um daqueles currículos, ainda inimagináveis e indizíveis, necessários e impossíveis, que nos impelirão, daqui para a frente, a curricularizar mais perigosamente. Por tudo isso, um pós-currículo da diferença é potente para fornecer outros pensamentos, sonhos, emoções, humanidades diferentes. Que tenhamos coragem, força e vontade ética para assumir os riscos e errâncias da produção de vários currículos da diferença. Currículos sem dogmas e sem certezas, que avançam abertos ao futuro como advento da justiça. Avançam em seu trabalho em processo, em suas estradas em andamento, em seus mares a fluírem. Currículos nos quais todos os dife-

Rev.Cad. Comun. Santa Maria, v.20, n.2, art 3, p.6 de 19, maio/ago.2016 
rentes que trabalhamos, caminhamos, navegamos, possamos, então, viver neles com mais singularidade e leveza, liberdade e beleza, dignidade e alegria. (CORAZZA, 2010, p.112-113)

O currículo, portanto, corresponde a uma construção social, com a organização e a estratificação própria das relações de poder (LOPES e MACEDO, 2010). Sabe-se que os currículos são feitos de escolhas, e nunca são um conjunto neutro de conhecimentos, mas resultado das seleções realizadas pelos cursos no sentido de eleger quais são legítimos, como afirma Apple (1994, p.59): "é produto de tensões, conflitos e concessões culturais, políticas e econômicas que organizam e desorganizam um povo".

A construção de novos currículos no ensino superior está vinculada, ainda, à compreensão da transformação do conhecimento universitário em pluriversitário. Para Santos (2005), a universidade tem um papel fundamental na resolução coletiva dos problemas sociais, auxiliando na construção do lugar do país dentro de um mundo globalizado, através da produção de conhecimento interdisciplinar, interativo, contextualizado, consumido e produzido por meio de novas tecnologias de comunicação e informação, e alterando as relações entre informação e conhecimento e formação e cidadania.

Nesse sentido, Almeida Filho (2011) afirma que a universidade brasileira está se recriando, com um projeto sintonizado com o desenvolvimento social, político, artístico e tecnológico da nação. Esta reforma universitária iniciou em 2008, amparada em seis eixos: expansão da graduação no território visando à inclusão social; recuperação do financiamento; ampliação do quadro de docentes; novos processos seletivos (Enem, SiSU); reestruturação da graduação; revisão da pós-graduação. A partir da referida reforma, emergiram novos modelos de ensino de graduação, com educação geral e regime de ciclos, em consonância com modelos curriculares dos países da Organização para Cooperação e Desenvolvimento Econômico (OCDE). Os modelos buscam a autonomia e diferenciam as trajetórias de formação profissional e acadêmica, baseando-se na valorização da inter/transdisciplinaridade, integrando saberes das artes e humanidades na pesquisa e formação; promovendo a etnodiversidade nos processos educacionais; formando sujeitos com inventividade e autonomia, preparados para cumprir a missão transformadora da universidade. Para o autor, "as categorias de etnodiversidade, interdisciplinaridade e autonomia constituem referências para pensar e agir frente a problemas complexos,

Rev.Cad.Comun. Santa Maria, v.20, n.2, art 3, p.7 de 19, maio/ago.2016 
estruturantes e emergentes na contemporaneidade" (ALMEIDA FILHO, 2011, p.5).

O Plano Nacional de Educação (PNE), sancionado no dia 25 de junho de 2014, que determinou metas, diretrizes e estratégias para a educação nos próximos dez anos, privilegiou a cultura como forma de despertar uma nova forma de educar, reconhecendo-a como uma política pública capaz de fomentar o acesso à cidadania, e considerando as escolas e a universidade como espaços de fruição, de produção e de acesso aos bens culturais. Para Domingues (2015, p.19), o objetivo é fazer da universidade e das escolas "espaços prioritários de promoção de competências criativas, produção e circulação da cultura brasileira, promovendo a aproximação entre o saber escolar e os saberes comunitários no currículo", contribuindo para que as identidades locais e regionais sejam consideradas a partir de uma contextualização cultural, social e histórica.

Sendo assim, a criação de um currículo para graduação em produção cultural, mostra essa nova posição da universidade na produção de um conhecimento pluriversitário, ou seja, de acordo com as necessidades de formação e capacitação atuais, permitindo o exercício do pensamento criativo em constante interação no processo de ensino e aprendizagem, estimulando a análise, investigação e reflexão de forma constante.

\section{A FORMAÇÃO EM PRODUÇÃO CULTURAL}

A formação dos profissionais da área da cultura, enquanto resultado do crescimento da educação superior e dos mercados artístico e literário, também contribuiu para a profissionalização desse campo.

A pesquisa intitulada "Mapeamento da Formação em organização da cultura no Brasil”, realizada em 2009, apontou a existência de nove cursos de graduação em Produção Cultural no Brasil: Bacharel em Produção e Política Cultural (Unipampa, campus Jaguarão); Ciências Sociais - Produção e Política Cultural (UCAM); Comunicação com Habilitação em Produção em Comunicação e Cultura (UFBA); Curso Superior em Ciências Sociais com concentração em Cultura e Sociedade (FGV); Engenharia de Produção com ênfase em Produção em Cultura (Unirio); Produção Cultural (UNEC); Produção Cultural (UFF); Produção e Marketing Cultural (UCAM); Relações Públicas com ênfase em Produção Cultural (Unipampa, campus São

Rev.Cad. Comun. Santa Maria, v.20, n.2, art 3, p.8 de 19, maio/ago.2016 
Borja $)^{8}$. Em 2014, foi criado o Curso de Comunicação Social com ênfase em Mídias Sociais ou Produção Cultural (UFPR), no Centro Acadêmico do Agreste, em Caruaru. Na referida pesquisa, Costa (2011), mapeou 257 instituições, 355 setores, 624 cursos e 98 publicações na área de organização da cultura. De acordo com o levantamento, $49 \%$ das instituições que trabaIhavam com formação em organização da cultura eram privadas, e apenas $29 \%$ eram públicas. Dos 624 cursos pesquisados, 75,88\% eram de extensão, com caráter mais esporádico, e, 9,49\% eram de especialização. Observando-se a pouca oferta de graduações na área: 0,96\%.

Com relação à profissionalização da área, a preocupação é constatada em documentos oficiais do Ministério da Cultura, como o Plano Nacional de Cultura, onde constam: aumento em $150 \%$ de cursos técnicos, habilitados pelo Ministério da Educação (MEC), no campo da arte e cultura com proporcional aumento de vagas; aumento em $200 \%$ de vagas de graduação e pós-graduação nas áreas do conhecimento relacionadas às linguagens artísticas, patrimônio cultural e demais áreas da cultura, com aumento proporcional do número de bolsas; 20 mil trabalhadores da cultura com saberes reconhecidos e certificados pelo Ministério da Educação (MEC); aumento em $100 \%$ no total de pessoas qualificadas anualmente em cursos, oficinas, fóruns e seminários com conteúdo de gestão cultural, linguagens artísticas, patrimônio cultural e demais áreas da cultura; aumento em 100\% no total de pessoas beneficiadas anualmente por ações de fomento à pesquisa, formação, produção e difusão do conhecimento; gestores de cultura e conselheiros capacitados em cursos promovidos ou certificados pelo Ministério da Cultura em 100\% das Unidades da Federação (UF) e 30\% dos municípios, dentre os quais, $100 \%$ dos que possuem mais de 100 mil habitantes e aumento de $95 \%$ no emprego formal do setor cultural.

Nas metas do Plano Nacional de Cultura, disponibilizadas em dezembro de 2011, foram previstas o aumento de trabalhadores da cultura com saberes certificados, refletindo a necessidade de estimular a formalização do mercado de trabalho do setor cultural, de modo a reduzir a informalidade do trabalho artístico, dos técnicos, produtores e demais agentes atuantes no campo da cultura. $O$ documento também destaca que a efer-

8 Dados do relatório final do "Mapeamento da formação e qualificação em organização cultural no Brasil”, coordenado por Antonio Albino Rubim, Alexandre Barbalho e Leonardo Costa. Disponível em: <http://www.organizacaocultural.ufba.br/>. Acesso em 10 de setembro de 2015 .

Rev.Cad.Comun. Santa Maria, v.20, n.2, art 3, p.9 de 19, maio/ago.2016 
vescência da economia da cultura e a expansão do mercado de trabalho colocam como fundamental a ampliação do conhecimento científico na área, primando pela formação de profissionais em nível universitário.

O aumento de trabalhadores da cultura também resultou na cooperação firmada entre os ministérios da Cultura e da Educação (Portaria Normativa Interministerial $n^{\circ} .1$, de 04 de outubro de 2007), que estabeleceram as diretrizes e critérios para a sua atuação, buscando a integração e a implantação de ações comuns e a consolidação de uma agenda bilateral entre os órgãos levando em consideração a interface entre PNC (Lei $n^{\circ}$. 12.343, de 2010) e PNE (Lei $n^{\circ} .10 .172$, de 2001), justificando a ampliação dos cursos de graduação na área.

De acordo com dados do Instituto Nacional de Estudos e Pesquisa Educacionais Anísio Teixeira (Inep), de 2002 a 2009, houve um aumento de $61 \%$ do número de vagas de graduação oferecidas nesse campo. Em 2009, existiam 4.432 cursos presenciais de graduação, nas áreas do conhecimento relacionadas às linguagens artísticas, patrimônio cultural, entre outras afins à cultura, que totalizaram 971.261 vagas.

Dessa forma, os cursos de produção cultural devem oferecer uma formação técnica/instrumental consistente, além de desenvolver um compromisso ético e político com a cultura e a sociedade, disponibilizando uma ampla informação cultural que permita a reflexão acerca da cultura e da sociedade (RUBIM, 2010).

\section{O PROFISSIONAL PRODUTOR CULTURAL}

Com relação às diferentes denominações acionadas para intitular o momento da organização da cultura e os profissionais responsáveis por seu tratamento, Rubim (2010) ressalta que: a denominação de gerentes e administradores culturais é predominante nos Estados Unidos e na França; a noção de animadores e promotores culturais possui tradição na Espanha; em vários países da América Latina, fala-se em trabalhadores culturais; e noutros países podem ser encontrados ainda termos como mediadores culturais, engenheiros culturais ou cientistas culturais (OEI, 1998, pp.19-20). Em Portugal, verifica-se a utilização da expressão programadores culturais para dar conta da esfera da organização da cultura (MADEIRA, 2002). Recentemente a noção de gestão cultural vem ganhando notoriedade em diversos países, inclusive ibero-americanos: "La noción

Rev.Cad. Comun. Santa Maria, v.20, n.2, art 3, p.10 de 19, maio/ago.2016 
de gestión cultural ingresa al discurso cultural en Iberoamérica con bastante influencia hacia la segunda mitad de la década de los ochenta, tanto en las instituciones gubernamentales como en los grupos culturales comunitários" (OEI, 1998, p.19) ${ }^{9}$.

Nesse sentido, a diversidade de termos indica a recente prática e estudos sobre a organização da cultura, e a denominação predominante no Brasil é a de produção cultural. Essa singularidade liga-se ao contexto histórico local e global:

A ausência de tradição na formação de gestores, a submissão da cultura à lógica de mercado e a fragilidade das políticas culturais do Estado nacional - fortemente acentuada nos oito anos de Fernando Henrique Cardoso e Francisco Weffort - são, por excelência, o contexto elucidativo da emergência da nomeação de produtores culturais, com as características que eles adquirem no país. A explicação elaborada ilumina igualmente a importância que o marketing cultural obteve no Brasil contemporâneo, quando se compara com outros países latino-americanos (RUBIM, 2008, p.53)

No que se refere à profissão, Villas Boas (2005) destaca que ela é emergente no Brasil e, por isso, exige um conjunto de conhecimentos e uma atuação crítica e reflexiva sobre a sua importância no desenvolvimento cultural, buscando a construção de um mercado cultural ético, diferenciado e eficiente, para que os projetos culturais sejam importantes e interessem aos autores, à mídia, aos patrocinadores e principalmente ao público, funcionando como elos obrigatórios dessa convergência de interesses. A autora não fala em produtor cultural, mas utiliza o termo gestor cultural, referindo-se ao profissional que deve conhecer a criação artística, ter sensibilidade e informação sobre as suas diversas linguagens, além de senso crítico sobre a diferença na atividade cultural proposta. Traz ainda, algumas características fundamentais ao perfil desse profissional, como: ser criativo e sensível, saber trabalhar em grupo, estabelecer metas, buscar informações, planejamento e acompanhamento, iniciativa, capacidade de correr riscos necessários, persistência, comprometimento, redes de contatos, entre outras. Além disso, elencou as funções do gestor como:

9 OEI - ORGANIZAÇÃO DOS ESTADOS IBERO-AMERICANOS. Cuadernos Cultura I. Conceptos básicos de administración y gestióncultural. Madrid: OEI, 1998.

Rev.Cad.Comun. Santa Maria, v.20, n.2, art 3, p.11 de 19, maio/ago.2016 
- Realizar novos estudos e pesquisa sobre o mercado cultural e suas interfaces;

- Fomentar o mercado cultural;

- Participar de redes de intercâmbio e informações;

- Promover a circulação e distribuição dos produtos culturais;

- Mobilizar, apoiar e motivar os artistas;

- Fidelizar o público através da formação de plateia;

- Resgatar e revitalizar as manifestações e ações culturais;

- Mobilizar e envolver a comunidade nesse processo de integração, resgate e sustentabilidade da cultura, numa perspectiva de não exclusão;

- Construir Planos de Desenvolvimento Cultural, nas organizações públicas e privadas, nos municípios, nos bairros, nas escolas;

- Viabilizar ações e projetos culturais;

- Registrar experiências através de publicações;

- Administrar espaços culturais. (VILLAS BOAS, 2005, p.108)

Ainda com relação à terminologia, Cunha (2007) utiliza o termo gestor cultural, mas afirma que não são permitidas definições precisas tendo em vista que as condições de comparação e análise mais profundas sobre a conceituação da expressão ainda são escassas. Nesse sentido, o gestor cultural exerce sua função ligada a cargos de direção, ou a frente de instituições públicas ou privadas do setor cultural, coordenando programas e projetos. De modo que

[...] o gestor cultural é aquele sujeito que: desenvolve e administra projetos culturais, desempenhando o papel de elo entre o artista e o Poder Público, a iniciativa privada e o público consumidor de cultura. Essa é a minha visão desse profissional, esteja ele trabalhando especificamente com alguma área (artística) ou mesmo trabalhando com a gestão de uma forma mais ampla, é ele quem desenvolve e administra projetos culturais (CUNHA, 2007, p.114).

Essa autora também tentou diferenciar o produtor do gestor cultural a partir de relatos de profissionais, caracterizando o produtor como um profissional mais executivo, e o gestor, como um profissional mais atuante estrategicamente. Mas destacou que, apesar de serem duas profissões diferentes, ambas se confundem no mercado cultural, em especial nos saberes desenvolvidos em cada uma, que coexistem no trabalho. Além das

Rev.Cad. Comun. Santa Maria, v.20, n.2, art 3, p.12 de 19, maio/ago.2016 
duas nomenclaturas, apontou a existência de outras como: mediadores, agentes e administradores culturais.

Rodrigues (2012), observando a profissionalização do setor, apontou que as diferentes terminologias provêm de diferentes momentos históricos, que dificultam o reconhecimento da profissão. Admite, ainda, que as três mais utilizadas sejam agente cultural, gestor cultural e produtor cultural, que nem sempre abrangem a realidade de atuação. No entanto, considera a gestão cultural como uma esfera mais ampla dos processos de mediação.

A profissão também derivou das mudanças ocorridas na cultura, com o surgimento do projeto cultural como forma de acesso aos recursos incentivados, uma nova organização "em que o espaço da gestão cultural floresceu e os conhecimentos sobre planejamento cultural e marketing cultural passaram a ser essenciais para os produtores e artistas" (MENDONÇA, 2012, p.210). Dentro dessa perspectiva, na década de 80, a função do produtor cultural era secundária na produção de cultura, pois as posições criativas eram mais valorizadas. A própria formação na área das artes é muito mais antiga no Brasil, portanto "toda uma tradição de ensino no campo das artes visuais, da música e das artes cênicas construiu uma notabilidade maior do artista sobre os meios de produção" (MENDONÇA, 2012, p.217).

A dificuldade de definir qual termo utilizar também se refere a um campo novo, pois: "pensar e planejar o campo da produção, circulação e consumo da cultura dentro de uma racionalidade administrativa é uma prática que pertence aos tempos contemporâneos" (CALABRE, 2008, p.66). Para Linda Rubim (2005, p.24), no Brasil, a opção mais usual para definir o profissional que trabalha com a organização da cultura é o produtor cultural, "mas a opção pelas expressões de uso mais corrente não garante que elas possam ser extensivas, ou suficientes, a todos os ramos da cultura". Mesmo com grande divergência com relação à denominação da profissão, Mendonça (2012) afirma que o produtor cultural é um termo "guarda-chuva", que compreende diversos fazeres profissionais e de pesquisa no campo da cultura.

Santos e Dourado (2014), destacaram a existência de diferentes perfis de produtores culturais resultantes das distintas atividades realizadas. Mas afirmaram que, apesar da existência de diferentes perfis, todos os profissionais promoviam a cultura a partir da elaboração ou gerenciamen-

Rev.Cad.Comun. Santa Maria, v.20, n.2, art 3, p.13 de 19, maio/ago.2016 
to de projetos culturais. Dentre os perfis observados, estavam: os produtores culturais envolvidos na elaboração de projetos culturais (voltados para editais públicos) e que, por vezes, produziam a carreira de artistas locais; os produtores que trabalhavam mais com eventos pontuais; os produtores que trabalhavam com a manutenção e gestão de coletivos culturais; e produtores que trabalhavam com a formação cultural, desenvolvendo ações voltadas para o desenvolvimento social por meio da cultura. Os diferentes perfis profissionais também estavam associados às potencialidades locais e regionais.

\section{CONSIDERAÇÕES}

A partir da exigência da sociedade por outros modos de gerir a cultura e comunicá-la, por meio das indústrias audiovisuais e das redes digitais, constituem-se diferentes perfis profissionais de produtores culturais. Os profissionais devem ter um perfil multidisciplinar, capaz de trabalhar com a incerteza, reposicionando-se no debate sobre a cultura, a identidade, a cidadania, a diversidade e a interculturalidade, compreendendo os vínculos entre criação, espetáculo, entretenimento e participação, e vislumbrando o sistema processual da cultura na sociedade. Como afirmou García Canclini (2008, p.361), profissionais "anfíbios", capazes de articular códigos e movimentos culturais das mais variadas procedências, mostrando que "é possível fundir as heranças culturais de uma sociedade, a reflexão crítica sobre seu sentido contemporâneo e os requisitos comunicacionais da difusão maciça". Um perfil profissional intercultural produzido pelas mais diversas hibridizações resultantes dos diferentes currículos dos cursos de graduação e das interferências do cenário social, capazes de trabalharem com a heterogeneidade e instabilidade das referências indenitárias das culturas contemporâneas e seus repertórios simbólicos.

Dessa forma, a construção desse perfil profissional concede maior responsabilidade para a formação universitária, responsável por produzir um conhecimento transdisciplinar, contextualizado, interativo, consumido e produzido por meio das novas tecnologias de comunicação e informação, alterando as relações entre informação e conhecimento, bem como entre formação e cidadania (SANTOS, 2005).

Para isso, é essencial que os cursos avaliem constantemente as suas matrizes curriculares no sentido de acompanhar as tendências do cená-

Rev.Cad. Comun. Santa Maria, v.20, n.2, art 2, p.14 de 19, maio/ago.2016 
rio social contemporâneo, e trabalhem na formação de aspectos como a sensibilidade para a diversidade cultural, o compromisso com a justiça e a equidade social, a criatividade, a iniciativa, a inovação, uma atitude proativa e a capacidade de organização, além de uma formação político-cultural global-local.

A partir do exposto, observa-se que o profissional produtor cultural tem fundamental importância no desenvolvimento da cultura, pois, a sua ação articuladora entre os sujeitos (artistas, mídia, público, patrocinadores) auxilia o crescimento da área cultural. Porém, os profissionais devem elevar o caráter educativo das ações culturais, contribuindo para o desenvolvimento pessoal e social dos indivíduos, transformando a realidade das comunidades e trabalhando a cultura como elemento do desenvolvimento. Além disso, as ações culturais devem destacar a diversidade brasileira, dar oportunidade às pequenas produções, mostrar ao público coisas diferentes e inovadoras, despertando-o para a arte e para a consciência cultural, garantindo acessibilidade, sustentabilidade e diversidade, pilares essenciais para o desenvolvimento da sociedade. 
CADERNOS DE COMUNICAÇÃO

UNIVERSIDADE FEDERAL DE SANTA MARIA

\section{REFERÊNCIAS}

ALMEIDA FILHO, Naomar de.; COUTINHO, Denise. Nova arquitetura curricular na universidade Brasileira. Ciencia e Cultura. [online]. São Paulo, vol.63, n.1, pp.4-5, 2011. ISSN 0009-6725.

ANASTASIOU, Léa das Graças Carmargos. A reorganização curricular: do currículo grade ao integrado. In: PEREIMA, Maurício José Lopes; COELHO, Elza Berger Salema (org.). Da proposta à ação: currículo integrado do curso de Medicina da UFSC. Florianópolis: Ed. da UFSC, 2005.

APPLE, Michael W. Ideologia e Currículo. São Paulo: Brasiliense, 1994.

BRASIL. Metas do Plano Nacional de Cultura. Brasília: MinC, 2011. Disponível em: <http://www.cultura.gov.br/documents/10883/11294/METAS_PNC_final.pdf/>. Acesso em maio de 2014 .

BRASIL. Cultura em números: anuário de estatísticas culturais.. 2 ed. Brasília: MinC, 2010. Disponível em: <http://culturadigital.br/ecocultminc/files/2010/06/Cultura-em-N\%C3\%BAmeros-web.pdf>. Acesso em 16 de abril de 2015.

BRASIL. Instituto Brasileiro de Geografia e Estatística - IBGE. Sistema de Informações e Indicadores Culturais: 2007-2010. Brasília: IBGE, 2013. Disponível em: <ftp://ftp.ibge. gov.br/Indicadores_Sociais/Sistema_de_Informacoes_e_Indicadores_Culturais/2010/ indic_culturais_2007_2010.pdf>. Acesso em maio de 2014.

BRASIL. Ministério da Educação e do Desporto. Catálogo Nacional de Cursos Superiores de Tecnologia. Brasília: MEC, 2010.

CALABRE, Lia. Profissionalização no campo da gestão pública da cultura nos municípios brasileiros: um quadro contemporâneo. In: Revista Observatório Itaú Cultural. São Paulo, nº 6, jul./set. 2008.

CORAZZA, Sandra Mara. Diferença pura de um pós-Currículo. In: LOPES, Alice Casimiro; MACEDO, Elizabeth (org.). Currículo: Debates contemporâneos. 3 ed. São Paulo: Cortez, 2010, p.103-114.

COSTA, Leonardo Figueiredo. Profissionalização da Organização da Cultura no Brasil: uma análise da formação em produção, gestão e políticas culturais. 2011. 238f. Tese

Rev.Cad. Comun. Santa Maria, v.20, n.2, art 3, p.16 de 19, maio/ago.2016 
(Doutorado em Cultura e Sociedade). Universidade Federal da Bahia, Faculdade de Comunicação, Bahia.

COSTA, Leonardo Figueiredo et al. Formação em Produção Cultural na UFBA: Uma Análise dos Alunos Egressos. UFBA: 2014. Disponível em: < https://repositorio.ufba.br/ ri/bitstream/ri/15672/1/monografia\%20PROUFBA.pdf>. Acesso em 17 de novembro de 2015.

CUNHA, Maria Helena. Gestão Cultural: profissão em formação. Belo Horizonte: Duo Editorial, 2007.

. Gestão Cultural. Coleção Políticas e Gestões Culturais. Bahia: P55 Edições, 2013. Disponível em: <https://conferenciadecultura.files.wordpress.com/2013/11/ cartilhas_secult_set13_gestc3a30_cultural_final.pdf>. Acesso em 15 de março de 2014.

DOMINGUES, Luis Paulo. Ensino Público e mercado: as concepções e os interesses que envolvem o Plano Nacional de Educação. Revista Caros Amigos: Especial Educação. São Paulo: 2015, ano XIX, nº 223, p.01-23.

FREIRE, Paulo. Pedagogia da autonomia: saberes necessários à prática educativa. São Paulo: Paz e Terra, 2011.

GARCÍA CANCLINI, Néstor. Culturas Híbridas: estratégias para entrar e sair da modernidade. 4 ed. São Paulo: Editora da Universidade de São Paulo, 2008.

. A globalização imaginada. São Paulo: Iluminuras, 2007b.

LOPES, Alice Casimiro. MACEDO, Elizabeth (org.). Currículo: Debates contemporâneos. 3 ed. São Paulo: Cortez, 2010.

MADEIRA, Cláudia. Os novos notáveis: os programadores culturais. Oeiras: Celta, 2002. MENDONÇA, Leandro José. O campo acadêmico da produção cultural - história e características. In: CALABRE, Lia (org.). Políticas culturais: pesquisa e formação. São Paulo: Itaú Cultural; Rio de Janeiro: Fundação Casa de Rui Barbosa, 2012. p. 205-219.

OEI - ORGANIZAÇÃO DOS ESTADOS IBERO-AMERICANOS. Cuadernos Cultura I. Conceptos básicos de administración y gestióncultural. Madrid: OEl, 1998

Rev.Cad.Comun. Santa Maria, v.20, n.2, art 3, p.17 de 19, maio/ago.2016 
CADERNOS DE COMUNICAÇÃO

UNIVERSIDADE FEDERAL DE SANTA MARIA

RODRIGUES, Luiz Augusto F. Formação e profissionalização do setor cultural. In: prag

MATIZES Revista Latino Americana de Estudos em Cultura. Ano 2, n.3, p.63-79, setembro de 2012. Disponível em: <http://www.pragmatizes.uff.br>. Acesso em 08 agosto de 2015 .

RUBIM, Antonio Albino Canelas. Singularidades da formação em organização da cultura no Brasil. In: Revista Organicom. Ano 7, N.13, p.37-38, 2010. Disponível em: < http://revistaorganicom.org.br/sistema/index.php/organicom/article/viewFile/313/359>. Acesso em setembro de 2014.

RUBIM, Linda. (org). Organização e Produção da Cultura. Salvador: EDUFBA, 2005.

SANTOS, Boaventura de Sousa. A universidade no século XXI: para uma reforma democrática e emancipatória da universidade. 2 ed. São Paulo: Cortez, 2005.

SANTOS, Elisabeth Cavalcante dos; DOURADO, Debora Coutinho Paschoal. Investidas do management no campo da cultura em Pernambuco: o caso dos produtores culturais. In: Cadernos EBAPE.BR. Rio de Janeiro, vol.12, n.1, Jan/Mar 2014. Disponível em: < http://www.scielo.br/scielo.php?pid=S1679-39512014000100011\&script=sci_arttext >. Acesso em 15 de abril de 2015.

Mapeamento da Formação em Organização Cultural no Brasil. Disponível em: <http:// www.organizacaocultural.ufba.br>. Acesso em outubro de 2014.

VEIGA, Ilma Passos Alencastro. Projeto político-pedagógico da escola: uma construção possível. 14 ed. São Paulo: Editora Papirus, 2002.

VILLAS BOAS, Rosa. Gestão Cultural. In: RUBIN, Linda (org.). Organização e Produção da Cultura. Salvador: EDUFBA; FACOM/CULT, 2005, p. 99-116. 


\section{Elisa Lübeck}

Possui graduação em Comunicação Social - Habilitação em Relações Públicas pela UFSM (2001), graduação em Sistemas de Informação pela UNIFRA (1998), mestrado em Extensão Rural pela UFSM (2004) e doutorado em Educação pela UNIJUí (2016). Atualmente é professora Adjunta do Curso de Relações Públicas - Ênfase em Produção Cultural da Universidade Federal do Pampa (UNIPAMPA), Campus São Borja-RS.

E-mail: elisaterra@unipampa.edu.br

\section{Maria Cristina Pansera-de-Araújo}

Possui graduação em Ciências Biológicas pela Universidade do Vale do Rio dos Sinos (1978), mestrado em Genética e Biologia Molecular pela Universidade Federal do Rio Grande do Sul (1981) e doutorado em Genética e Biologia Molecular pela Universidade Federal do Rio Grande do Sul (1997). Atualmente é professora titular da Universidade Regional do Noroeste do Estado do Rio Grande do Sul, Departamento de Ciências da Vida e no Programa de Pós-Graduação em Educação nas Ciências.

E-mail: pansera@unijui.edu.br

RECEBIDO EM: 06/06/2016

ACEITO PARA PUBLICAÇÃO: 15/07/2016 